\title{
Centralising the Admission Process in a German Hospital
}

\author{
Melanie Reuter-Oppermann, \\ Laura Kienzle, Anne Zander \\ Karlsruhe Service Research Institute, \\ Karlsruhe Institute of Technology \\ \{melanie.reuter, laura.kienzle, anne.zander\}@kit.edu
}

\author{
Diana Buchmüller, \\ David Goldberg \\ Städtisches Klinikum Karlsruhe \\ diana.buchmueller@klinikum-karlsruhe.de \\ mail@dr-goldberg.com
}

\begin{abstract}
The admission process is the first part of a hospital stay for all in- and outpatients. For the hospital, it is a critical part as all the necessary data must be stored in the hospital information system and all legal documents have to be signed in order to guarantee the best possible treatment and efficient logistics. In this paper, we focus on the admission process in a German hospital. As the admission of a patient, especially informing and advising him, is very time consuming, the hospital aims at installing a digital admission process. The first important step towards this goal is pooling the current decentralised patient admission in one new building. Using a simulation, we determine efficient staffing levels and rosters for the centralised admission and analyse the expected waiting times for the patients. In addition, we outline potential steps towards a digital patient admission process.
\end{abstract}

\section{Introduction}

Hospitals in Germany, similar to many other countries worldwide, face numerous challenges today. They have to deal with an increasing cost pressure forcing the hospitals to improve the efficiency of all their processes, medical as well as logistical. On the other hand, patients can usually choose the hospital for their treatments and expect increasing service levels.

The admission of patients when they enter the hospital in order to receive treatment is a critical part of the overall process, as all the necessary information of the patients needs to be entered into the Hospital Information System (HIS). This is not only important to ensure the right treatments, but it is also crucial for issuing invoices to the insurance companies once a patient has left the hospital. If the data is faulty or not all documents are signed - as it especially happens for emergency patients that go directly to the emergency department -, bills are not paid by the German insurance companies and hospitals loose significant amounts of money.
Currently, the admission of elective patients, inpatients as well as outpatients, is done by nurses and the data insertion happens manually in most hospitals, while most HIS do not assist a user by showing the desired data format or alert the user of inconsistencies. We exclude emergencies here as these patients will always enter the Emergency Department directly and will be admitted there. Often, the admission of a patient takes a lot of time as nurses need to explain the legal documents patients must sign and offer extra services that a patient needs to decide about spontaneously. In addition, all patients are usually asked to come in at the same time - the beginning of the nurses' shift - which means that the majority has to wait until they are served and the waiting room is crowded for a long time.

Also, as nurses perform the admissions, their workforce cannot be used for actually nursing tasks and is therefore missing in the hospital. Moreover, the nurses would also prefer to work in their actual wards helping patients instead of inserting data. Alternative personnel is usually not available for this task.

It is apparent that the current process offers potential for significant improvements, in the logistics in general, but also the support by Information Systems (IS).

In this paper, we study the admission process in a hospital in Karlsruhe, Germany. While the aim is to digitalise the admission process in the future, the first step is to centralise it. Accordingly, we discuss the restructuring of the admission process and present a centralised outline. Using Analytics and Operations Research (OR) we determine staffing levels for the admission process and simulate the utilisation. Furthermore, we discuss steps towards the digital patient admissions.

The remainder of the paper is structured as follows. In Section 2 we present the relevant literature from an IS and an OR perspective. Section 3 presents the case study for the Städtisches Klinikum Karlsruhe (SKK) in Germany while Section 4 discusses a future digital admission process. The paper closes with a conclusion and an outlook in Section 5. 


\section{Foundations and Related Work}

In this section, the relevant literature from both disciplines, Operations Research as well as Information Systems, is presented. The literature distinguishes between the medical and the administrative admission [21]. In this work we only focus on the latter. The literature on the actual admission process and the use of IS for it is comparably scarce, while many publications exist on topics around the actual admission. Therefore, we present the relevant topics in this section and give a brief overview over existing work.

\subsection{Operations Research Literature}

Hutzschenreuter et al. apply the idea of centralisation to the admission scheduling problem [11]. Instead of every clinic scheduling patient admissions individually these are determined hospital wide, in order to prevent unforeseen bottlenecks and shortages. They use an agent-based simulation model to analyse the patient mix. Min and Yih use a simulation to study different queue disciplines for patient registration in an outpatient clinic [15]. They consider factors like noshows, patient punctuality and appointment blocks in their study. Mocarzel et al. propose a simulation model to analyse and improve several issues regarding the admission process and resource workflow faced by a hospital [16].

Other papers tackle problems arising around the patient admission process. Sundaramoorthi et al. propose a simulation-based optimisation approach for assigning nurses to newly-admitted patients in the respective clinics with the aim of balancing the nurses workload [22]. The patient admission scheduling problem determines the days when patients are to be admitted to the hospital, minimising the waiting times for the patients and maximising the hospital utilisation, with the restriction that only limited resources (e.g. nurses, operating theatres) are available. Lowery uses a simulation model to address the problem taking all patient admissions and discharges into account [13]. In an earlier publication Kolear uses queueing approaches and presents a Markovian model for the hospital admission scheduling problem [12].

Determining staffing levels is a classical OR problem, inside and outside health care, for example with an application to call centres as in [3]. Nurse rostering is a challenge for the majority of hospitals. Reviews on operations research approaches can be found in [4] and [6]. An overview over discrete-event simulations applied to health care problems with a focus on hospitals is presented in [9].
The „9 am phenomenon“, meaning that patients are all arriving between 8 am and 9 am on the day of admission, leads to an increased workload and stress for the nurses and long waiting times for the patients [21]. It can be prevented by spreading patient arrivals over the time horizon when assigning appointments to patients. Reviews on appointment planning can be found in [5], [10] and [2].

\subsection{Information Systems Literature}

Hospital information systems (HIS) are crucial for all medical and logistical processes nowadays, promising to improve quality of care and the efficiency of processes. The main challenges for the use of IS in health care are privacy concerns, interoperability, and resistance to change [7], [20]. Unfortunately, the health care domain is characterised by low and slow adoption of IS [1], even though the integration of sophisticated information systems could have a significant impact. User assistance and user assistance components like conversational agents or digital personal assistants can help users performing their tasks better [14]. Morana et al. start addressing the question on how to design user assistance components for health care information systems in order to improve the user's performance [17]. Morana et al. propose a taxonomy of guidance design features to describe the provided guidance along ten dimensions [18]. Chatbots are a promising new technology that could be helpful for the digital admission in the future [8].

\section{Patient Admission in the SKK hospital}

In this section we consider the patient admission process at the hospital „Städtisches Klinikum Karlsruhe" (SKK) in Karlsruhe, Germany. The SKK is a hospital with maximum medical care with 1536 beds. Each year, 63,000 inpatients and 185,000 outpatients are treated. In the past, each clinic within the hospital has organised its admission individually. With the construction of a new main hospital building, the admission of all elective patients shall be merged and executed in a new location on the ground floor of this building.

Figure 1 shows the hospital campus and the relevant clinics / buildings for this project (left part of the figure). Their patients shall all be admitted in the new central building „M“ (right part of the figure).

In the envisioned admission process, patients enter the lobby of building $M$ where they are served by nurses at several service desks. If all desks are occupied when a patient enters the building, he can sit down in the waiting area and wait for the next nurse to become 
available. Service desks are separated to ensure the patient's privacy.

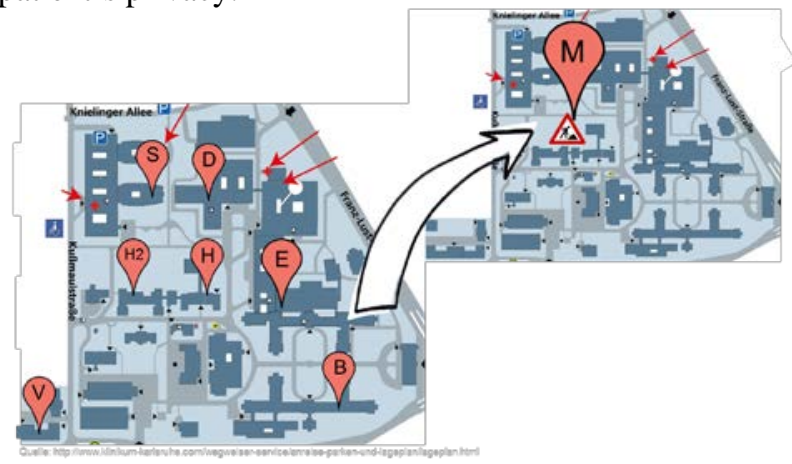

Figure 1. SKK campus

A centralised admission will be more efficient than a decentralised process, as it allows to use dedicated staff for the task instead of risking that untrained nurses admit patients in their respective clinic, when the nurse that is usually in charge is not available. In addition, pooling resources and centralising the process figuratively means combining several queues into one. Research has shown that this is beneficial regarding the number of necessary servers as well as waiting times (see for example [19]).

During the admission itself, the patient's insurance card is inserted into a reader and data is transferred to the HIS, Agfa Orbis. Already stored data from potential earlier visits as well as new data is verified by the patient and the nurse asks for additional information, e.g. if the referring general practitioner is to be informed about the results. Then, the patient is informed about additional services that he can choose and the occurring costs. These services can be split into medical and nonmedical services. An example for a medical service is treatment by a chief physician, non-medical services include single or double rooms, televisions, robes or newspapers. All those services are not paid by the public insurance. If a patient wants to book these services, a contract must be signed and the patient needs to pay for those services himself. Also, patients must pay $10 €$ per day (for at most 28 days per year) when being treated in a hospital. In certain cases, patients are exempted from this fee, which they have to prove during the admission. Together with the treatment documents, a patient's signature is necessary at least 10 times. At the end, the patient receives a folder with the hospital's terms and conditions as well as all relevant information.

\subsection{Input Data}

In Germany, two types of insurances exist, public and private insurance. The type of insurance has a significant impact on the admission of a patient, as depending on the type different documents must be signed and additional services are offered. At SKK, 13\% of the patients were privately insured, $87 \%$ publicly. While these numbers present average values and can obviously vary from day to day, the daily percentages could not be determined. Therefore, the average values were used for the case study. On average, the admission of a privately insured patient takes 15 minutes, while only 10 minutes are assumed when a patient is a member of a public insurance company.

For the staff schedules either no restrictions or 4 and 8 hour blocks were assumed.

We consider two scenarios with two stages each. The scenarios differ in the number of centralised clinics: In scenario 1 only the large clinics are centralised, i.e. clinics B, D, E, H, V, and in scenario 2 all clinics. The stages include only inpatients (stage 1 ) or inpatients and outpatients (stage 2). This leaves us with four configurations to test, with the fourth (scenario 2 stage 2 ) is the one desired by SKK. The other three cases can be interpreted as potential interim solutions. The percentages of patients admitted to each clinic are displayed in Table 1.

\section{Table 1. Patients per clinic}

\begin{tabular}{|l|c|}
\hline Clinic & \% of patients \\
\hline Clinic B & 10 \\
\hline Clinic D & 10 \\
\hline Clinic E & 25 \\
\hline Clinic H & 4 \\
\hline Clinic H2 & 22 \\
\hline Clinic V & 26 \\
\hline Decentralised ward & 2 \\
\hline RCPU & 1 \\
\hline
\end{tabular}

As a data source we used the information from Agfa Orbis, the HIS used in the SKK hospital. Data was available for inpatients and outpatients of 2015 and the first half of 2016. We clustered the data for each clinic and patient type and used this to form the input for the four cases (two scenarios and two stages).

Interviews with staff in clinics $\mathrm{B}$ and $\mathrm{E}$ gave additional insights into the process, the current challenges and the recorded data.

We evaluate the four cases described above according to the following measures:

- Patient arrival rate / week

- Number of necessary service stations

- Number of necessary staff and staff rosters

- Additional walking distances for the patients

- Additional number of walks for the patients 


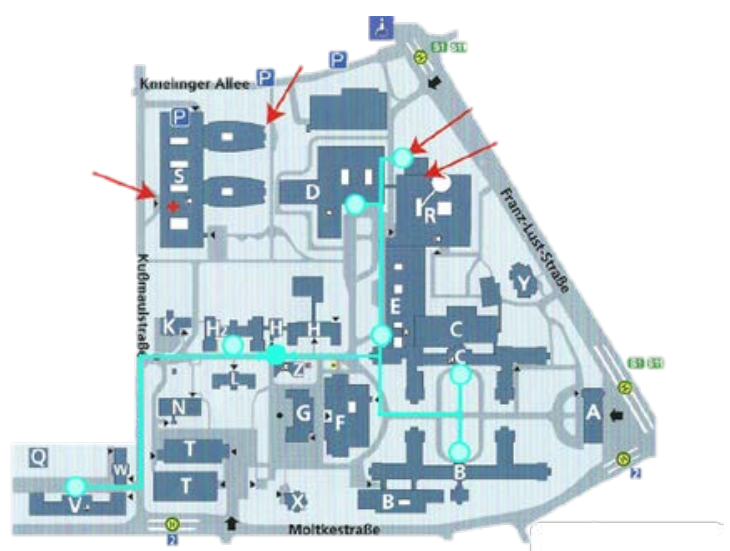

Figure 2. Pathways on the SKK campus

Figure 2 shows the ways patients need to walk from the new building $\mathrm{M}$ to their designated clinic for those included in scenario 1 . The distances per walk are summarised in Table 2.

Table 2. Distances to the clinics

\begin{tabular}{|l|c|c|}
\hline Clinic & Distance (m) & Patients (\%) \\
\hline B & 266 & 21 \\
\hline D & 266 & 17 \\
\hline E & 190 & 39 \\
\hline H & 0 & 11 \\
\hline V & 276 & 12 \\
\hline
\end{tabular}

These distances lead to average walking distances of 207 meters for a patient in scenario 1 and 229 meters in scenario 2. For many patients this will not be a problem as nowadays they also have to walk some distance, for example when they arrive by public transport or when

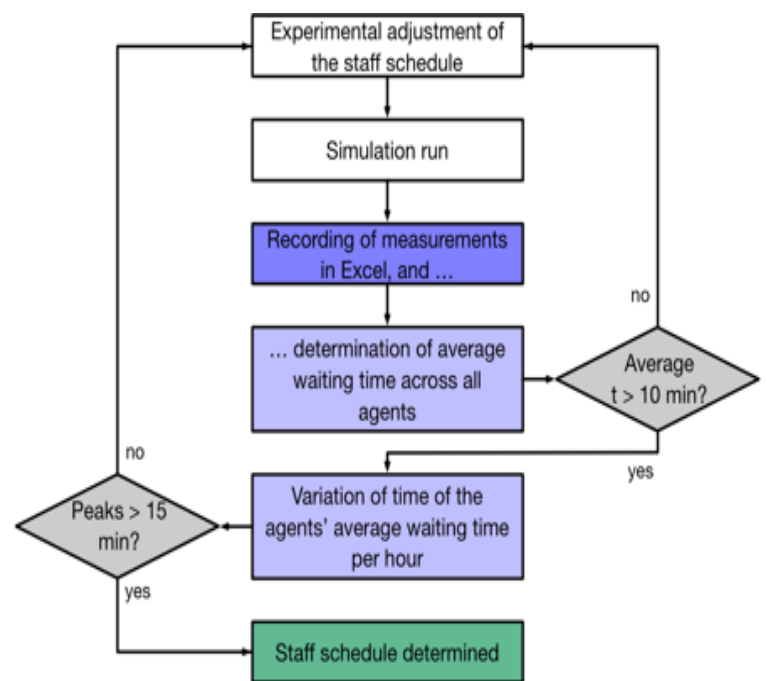

Figure 3. Optimisation flow chart they are dropped off at one of the official parking lots. The hospital must keep in mind, though, that it might be necessary to transport some of the patients to their dedicated clinic after the admission, if they cannot walk there on their own, leading to a workload increase for the transport service staff.

\subsection{Simulation and Optimisation}

We use simulation to analyse possible staff schedules and to determine the one with the fewest staff demand that ensures a waiting time of less than 10 minutes on average for the patients. In order to do that, we follow the optimisation process displayed in Figure 3.

We start with a staff schedule, run the simulation and determine the waiting times. If the average is over 10 minutes, we have a closed look at the waiting time distribution over the time horizon. If the maximum waiting times are smaller than 15 minutes, we have found our staff schedule. If they exceed the limit, the staff schedule is adapted by stepwise increasing the necessary staff numbers for exceeding hours. In addition, the staff utilisation is analysed. If the utilisation is close to $100 \%$, the staff numbers are increased, too. In case the utilisation is very small, the numbers are decreased. Also, if the average waiting time is a lot smaller than 10 minutes, staff numbers are decreased, in order to safe workforce for the hospital, for cost reasons, obviously, but also because it is challenging to hire qualified medical personal at the moment and available workforce must be used very efficiently. Then, the simulation is run again. This leads

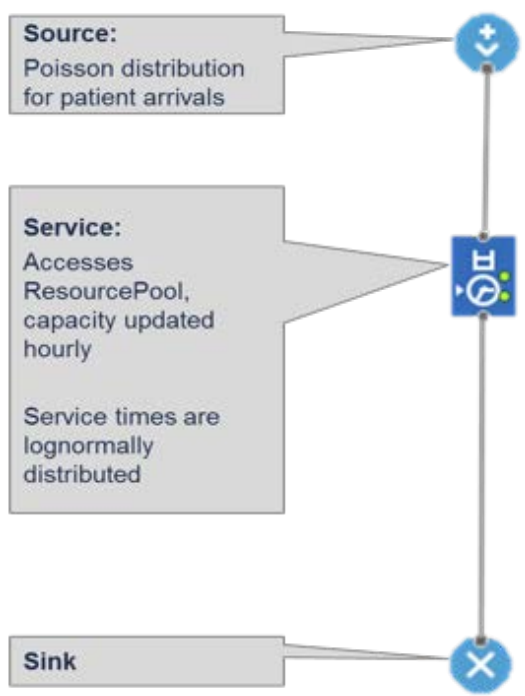

Figure 4. Simulation outline 
to a staff demand per hour for each of the four configurations.

The simulation resembles a simple queuing model and consists of a source representing the patients, a service/resource pool for the actual admission and a sink meaning that patients leave the queue after they have been served. The main structure is displayed in Figure 4. The capacity of the service node is updated hourly, allowing for varying numbers of nurses to admit patients. The service times follow a lognormal distribution.

The simulation is implemented in the simulation software AnyLogic. For verification purposes we have compared the arrival rates with the actual simulated arrival rates leading to a deviation of less than $2 \%$ which we assume to be acceptable. We have also checked that the simulated service time distribution resembles the lognormal input distribution. In addition, we have validated the simulation with responsible SKK employees. At discrete time events, patients enter the system. An insurance type (public or private) is assigned to the patient with probabilities of $87 \%$ for public and and $13 \%$ for private insurance, respectively. Then, the patient enters the service area corresponding to the patient entering the hospital. If a server is available, he is served on average $10 / 15$ minutes with the service time following a lognormal distribution. The simulation flow chart is summarised in Figure 5.

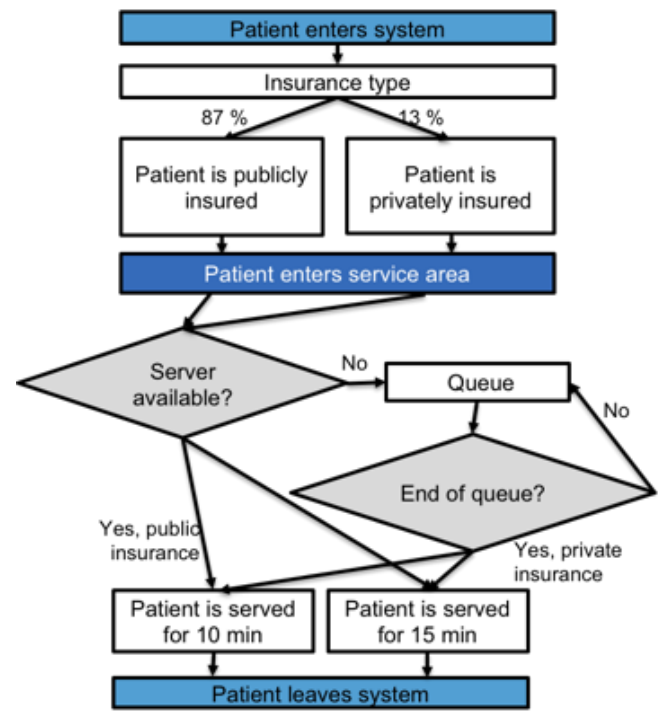

Figure 5. Simulation flow chart

Based on the hourly demand of staff, a staff roster is determined using a simple optimisation model which can be solved with a solver like IBM ILOG CPLEX. In the model the parameter $b_{i}$ represents the minimum number of nurses that are needed at time i, with i going from 1 to 24, resembling the hours of a day. Note that 24:00 is equal to 0:00. Only two decision variables, $\mathrm{x}_{\mathrm{i}}{ }^{\mathrm{a}}$ and $\mathrm{x}_{\mathrm{i}}^{\mathrm{b}}$, are necessary for the model. They define the number of nurses that start a shift at time $i$, with a denoting the 8-hour shifts and b the 4-hour shifts. The objective function minimises the shift starts and therefore the number of nurses. In order to prefer 18 hour shift to 2 consecutive 4-hour shifts in order to safe personnel, the decision variables $\mathrm{x}_{\mathrm{i}}^{\mathrm{b}}$ are weighted with the factor 1.99 in the objective function. The constraints ensure that the demand for nurses is fulfilled in every hour of the day.

$$
\begin{aligned}
& \min \sum_{i=1}^{24}\left(1.99 \cdot x_{i}^{a}+x_{i}^{b}\right) \\
& \sum_{i=21}^{24} x_{i}^{b}+\sum_{i=17}^{24} x_{i}^{a} \geq b_{24} \\
& \sum_{i=22}^{24} x_{i}^{b}+x_{1}^{b}+\sum_{i=18}^{24} x_{i}^{a}+x_{1}^{a} \geq b_{1} \\
& \sum_{i=23}^{24} x_{i}^{b}+\sum_{i=1}^{2} x_{i}^{b}+\sum_{i=19}^{24} x_{i}^{a}+\sum_{i=1}^{2} x_{i}^{a} \geq b_{2} \\
& \ldots \sum_{i=20}^{23} x_{i}^{b}+\sum_{i=16}^{23} x_{i}^{a} \geq b_{23} \\
& \forall i: x_{i}^{a}, x_{i}^{b} \in \mathbb{N}^{+}
\end{aligned}
$$

Table 3. Summary of the results

\begin{tabular}{|l|c|c|c|c|}
\hline \multirow{2}{*}{} & \multicolumn{2}{|c|}{ Scenario 1 } & \multicolumn{2}{c|}{ Scenario 2 } \\
\cline { 2 - 5 } & Stage 1 & Stage 2 & Stage 1 & Stage 2 \\
\hline $\begin{array}{l}\text { Patients/ } \\
\text { week }\end{array}$ & 576 & 1150 & 654 & 1544 \\
\hline $\begin{array}{l}\text { Add. } \\
\text { walks/ } \\
\text { week }\end{array}$ & 513 & 1081 & 589 & 1482 \\
\hline $\begin{array}{l}\text { Add. } \\
\text { distance/ } \\
\text { week } \\
\text { (km) }\end{array}$ & 119 & 263 & 131 & 292 \\
\hline $\begin{array}{l}\text { Servers/ } \\
\text { day }\end{array}$ & 7 & 10 & 7 & 14 \\
\hline
\end{tabular}

\subsection{Results}

An overview over the simulation results can be found in Table 3 . The number of patients considered increases from scenario 1 to scenario 2, but it is even more significant between stage 1 and stage 2 . That is, in scenario 1 stage 1 , only 576 patients are admitted to the hospital per week, while in scenario 2 stage 21544 
patients would need to be served in the new building. It is clear that if the admission is centralised in one building where the majority of the patients are not treated, they need to first go to this building and then to their actual clinic.

The most important information for the hospital are the numbers of nurses that are necessary for serving the patients for the four cases. For scenario 2 stage 214 nurses are necessary. At stage 1 for both scenarios 7 nurses are sufficient. For stage 2 of scenario 1 we have calculated 10 nurses to be sufficient.

Table 4. Simulation results

\begin{tabular}{|l|c|c|c|c|}
\hline \multirow{2}{*}{} & \multicolumn{2}{|c|}{ Scenario 1 } & \multicolumn{2}{c|}{ Scenario 2 } \\
\cline { 2 - 5 } & Stage 1 & Stage 2 & Stage 1 & Stage 2 \\
\hline $\begin{array}{l}\text { Average } \\
\text { waiting } \\
\text { times } \\
\text { (min) }\end{array}$ & 5.034 & 5.774 & 4.986 & 4.6368 \\
\hline $\begin{array}{l}\text { Waiting } \\
\text { times for } \\
\text { 90 \% } \\
\text { (min) }\end{array}$ & $<15$ & $<19$ & $<15$ & $<12$ \\
\hline $\begin{array}{l}\text { Average } \\
\text { queue } \\
\text { length }\end{array}$ & 2.502 & 4.403 & 2.767 & 4.514 \\
\hline
\end{tabular}

Table 4 displays the simulation results in more detail. The average waiting time for all four cases is less than 6 minutes for scenario 1 and less than 5 minutes for scenario $2.90 \%$ of the patients need to wait less than 15 minutes for stage 1 or 19 / 12 minutes for stage 2 in scenario 1 / scenario 2 . In average, 2.5 to 4.5 patients are waiting in the queue.

\section{Number of nurses per day}

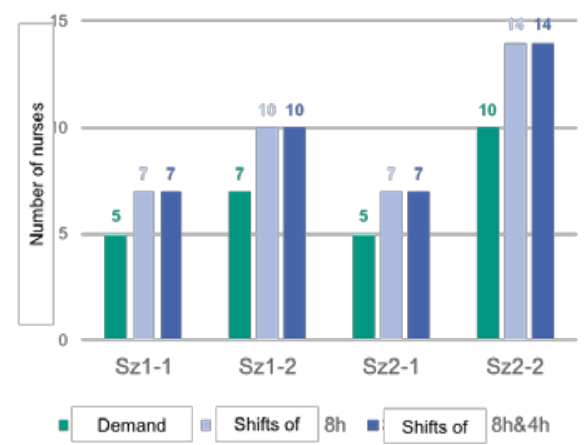

Figure 6. Number of nurses

Figures 6 and 7 show the number of nurses and of working hours per day, for the calculated demand and the corresponding rosters, assuming only 8-hour and 4/8-hour shifts. It becomes apparent that in both cases more nurses and working hours are necessary. While the number of nurses is the same, allowing for 4-hour shifts decreases the amount of working hours by $14.3-15 \%$.

We ran a test with a decreased average service time for privately insured patients of 12 minutes. Due to the small percentage of those patients, it did not decrease the number of nurses, but the number of working hours

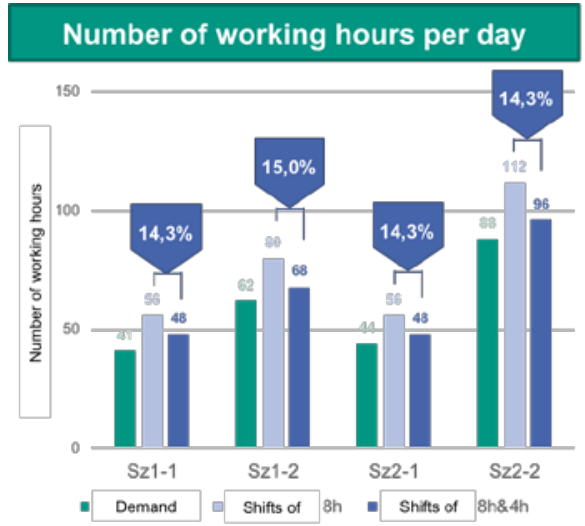

Figure 7. Working hours

by $14.3 \%$. A service time decrease for publicly insured patients would have a significantly larger impact, but is hard to achieve in practice without digitalising the admission process.

In a second part of the sensitivity analysis we have changed the patient arrivals to be uniformly distributed, to analyse the effect of using appointments. Then, the number of nurses can be reduced from 14 to 9 for the scenario 2 stage 2 case, and the number of working hours can be decreased by nearly 50 \% from 112 to 68 hours.

The corresponding distributions of the waiting times and the number of nurses per hour are shown in Figures 8 - 10 for all four combinations of scenarios and stages. Figure 8 shows the determined demands for nurses per hour. Accordingly, Figure 9 tells the number of nurses starting a shift in each hour, assuming only 8-hour shifts are possible. Accordingly, when determining the results shown in Figure 10 also 4-hour shifts were possible. It is apparent that the waiting time distributions differ for the cases and the possible shift types. 


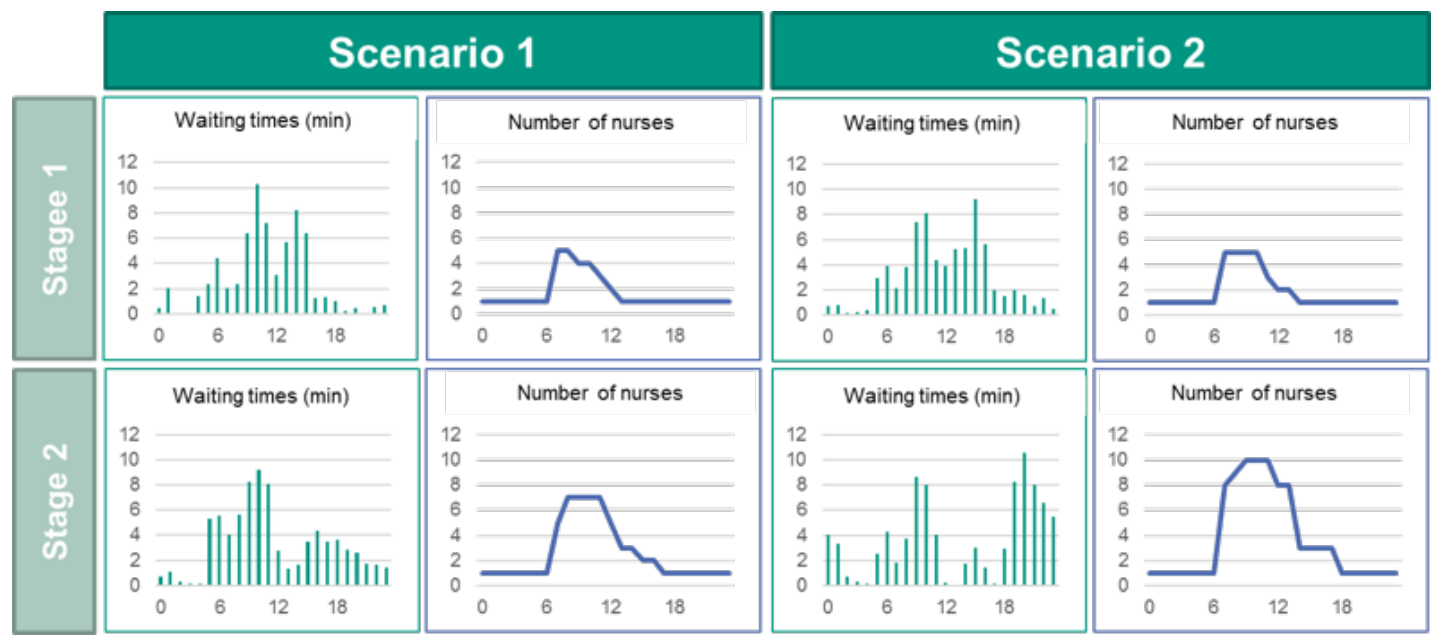

Figure 8. Results for the nurse demands

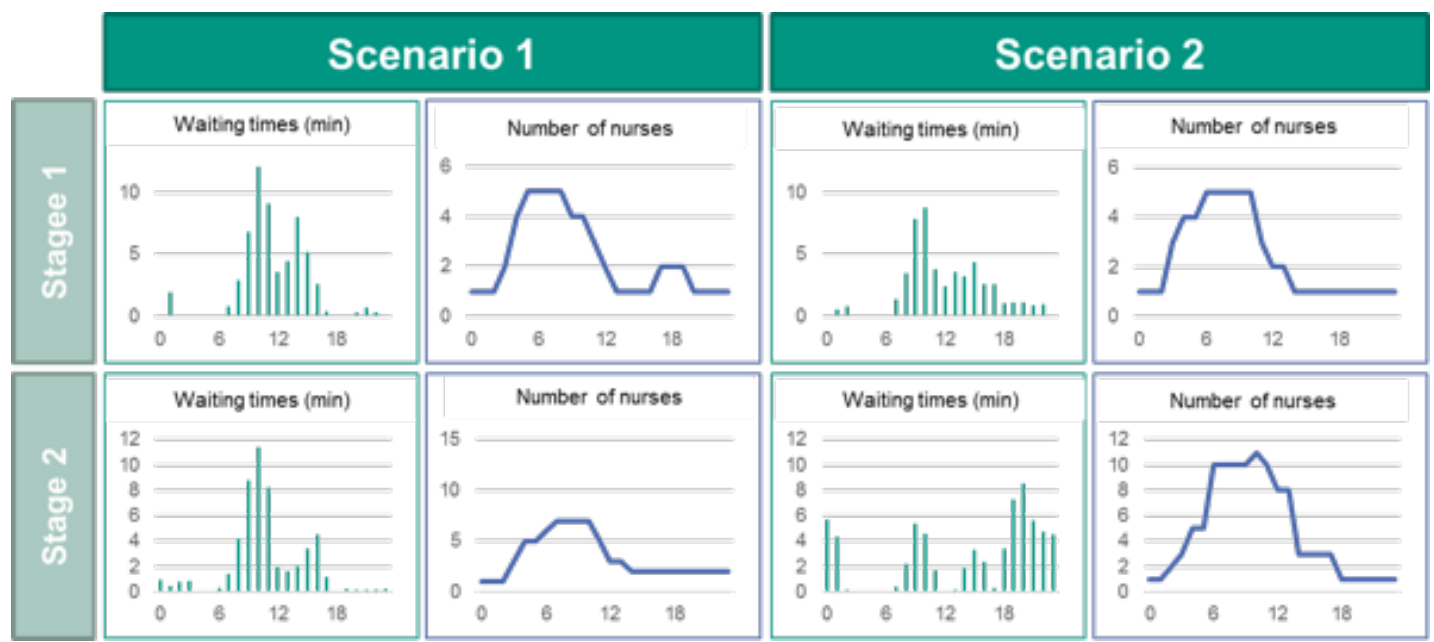

Figure 9. Results for 8-hour shift schedules

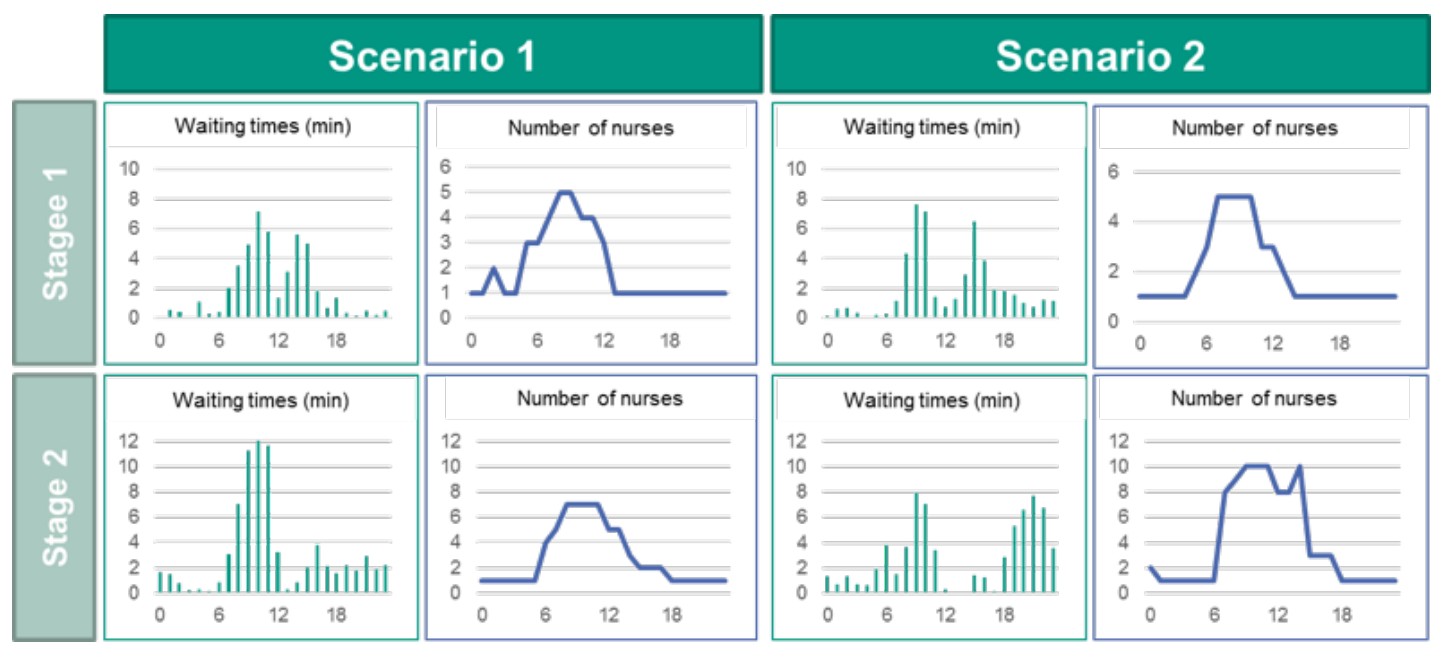

Figure 10. Results with 4- and 8-hour shifts 


\section{Towards a Digital Admission Process}

In an ideal admission process elective patients insert all the relevant data and read and sign the legal documents before entering the hospital. That is, when the decision is made that a patient needs treatment in a hospital, the process starts and the patient gets access to an IS. He has then time to review the documents, enter the data and decide on extras or upgrades like a single room. This also allows the patients to consult their relatives and ask for their opinion or their help. They can do it in their own time and whenever it suits them best.

A sophisticated IS assists the user when inserting the information. This includes displaying and checking the right data format, ensuring consistency and explaining possible options. That is, the data insertion is decoupled from the physical admission of the patient.

One can think of several intermediate stages between the current manual on site and the decoupled digital admission process. A first stage would mean that the legal documents are handed to a patient before the treatment, ideally sent by email or just available on a hospital website or in a hospital app. This would already decouple one part of the very enduring admission process, as especially elderly patients have enough time to read the legal documents, if necessary together with the relatives. In the hospital, nurses would still need to transfer the data into the HIS, though.

Therefore, stage 2 contains the idea of installing terminals in the admission area that patients can use to enter their data themselves. The installed IS should also include user assistance, maybe be even based on an interactive chatbot giving the patient the impression that he is still served by hospital personnel. While these terminals will reduce the nurses' workload for the admissions, there will still be the need for them to admit (elderly) patients that are put off by the terminals and need personal assistance.

At stage 3 , the admission process is completely digital and decoupled from patients physically entering the hospital as described above. Then, few nurses might still be necessary to serve patients that forgot to complete the admission process or that have questions that could not be answered beforehand, but the workload will be significantly lower and the process more efficient.

\section{Conclusions and Outlook}

In this paper we have studied the hospital admission process with focus on a centralised process. We have presented a mathematical approach to determine appropriate staffing levels and tested it with data from the SKK hospital in Karlsruhe, Germany. The results show that the service times have a big influence on the solutions. This means that on the one hand it is very important that these are assumed correctly and on the other if they can be reduced, this will have a significant impact on the nurses' workload. Regarding the shift times, 4-hour blocks lead to a smaller number of necessary staff as the admissions are not uniformly distributed over the 8-hour working day, but peaks occur only for a few hours. If patient arrivals are spread over the time by giving them individual appointments the number of necessary staff can again be decreased significantly. Using OR techniques the appointment times can be determined [5]. In addition, we have discussed the digitalisation of the patient admission process.

As future work, we plan on designing and implementing a fully digital patient admission process at the SKK that also integrates the patient's discharge in such a way that the process, i.e. the preparation of discharge documents, the invoice etc., is already started when the patient enters the hospital. That means that the overall documentation process of a patient is consolidated increasing the efficiency and decreasing the possibility for errors. One main challenge will be the training and preparing of nurses and staff accordingly, as to the changed processes as well as their adapted working tasks. Efficient communication will play an important role in the envisioned admission process. Even with a digital process, empathy and knowledge of human nature will still be very important.

From an Operations Research point of view, the admission process could be modelled as a queueing model and combined with the simulation to a simulation-optimisation approach, iteratively determining the efficient staffing levels and rosters, similar to our approach presented in this study. While an integrated approach would lead to similar results, it would be more convenient to use for hospitals as they only have to run one model.

Studying the admission process in practice has shown that the expected service times (10 / 15 minutes) is too short to explain all documents and additional services in detail. Therefore, it is important to send information to the patient beforehand, as otherwise the process could be delayed or the hospital could lose money, its patients are not booking the services or insurance companies are covering the treatment costs.

In addition, the design of an appropriate IS with assistance functionality for the patients, relatives and staff is another important topic for future research.

We will also be able to use the simulation to analyse the impact of appointments or even shorter admission times when patients enter their data beforehand, but need a short central admission for legal reasons anyway. The simulation can also be used to analyse the use of 
terminals in order to determine if an installation would be beneficial for the hospital and how many terminals would be necessary.

\section{References}

[1] J. Adler-Milstein, and D.W. Bates, "Paperless healthcare. Progress and challenges of an IT-enabled healthcare system", Business Horizons, Vol. 53 No. 2, 2010, pp. 119-130.

[2] A. Ahmadi-javid, Z. Jalali, and K.J. Klassen, “Outpatient Appointment Systems in Healthcare: A Review of Optimization Studies”. European Journal of Operational Research, 2016.

[3] J. Atlason, M.A. Epelman, and S.G. Henderson, "Call Center Staffing with Simulation and Cutting Plane Methods”, Annals of Operations Research, 2004, pp. 127-333.

[4] E.K. Burke, P. De Causmaecker, G.V. Berghe, and H. Van Landeghem, "The state of the art of nurse rostering“, Journal of scheduling, 7(6), 2004, pp. 441-499.

[5] T. Cayirli, and E. Veral, "Outpatient Scheduling in Health Care: a Review of Literature", Production and Operations Management, 12(4), 2009, 519-549.

[6] B. Cheang, H. Li, A. Lim, and B. Rodrigues, "Nurse rostering problems-a bibliographic survey”, European Journal of Operational Research, 151(3), 2003, pp. 447-460.

[7] M.W. Chiasson, and E. Davidson, "Pushing the contextual envelope. Developing and diffusing IS theory for health information systems research", Information and Organization, Vol. 14 No. 3, 2004, pp. 155-188.

[8] U. Gnewuch, S. Morana, and A. Maedche, “Towards Designing Cooperative and Social Conversational Agents for Customer Service”, In Proceedings of the International Conference on Information Systems (ICIS) 2017.

[9] M.M. Günal, and M. Pidd, "Discrete event simulation for performance modelling in health care: a review of the literature”, Journal of Simulation, 4(1), 2010, pp. 42-51.

[10] D. Gupta, and B. Denton, “Appointment scheduling in health care: Challenges and opportunities”, IIE Transactions, 40(9), 2008, pp. 800-819.

[11] A.K. Hutzschenreuter, P.A. Bosman, I. Blonk-Altena, J. van Aarle, and H. La Poutré, "Agent-based patient admission scheduling in hospitals", In Proceedings of the 7th international joint conference on Autonomous agents and multiagent systems: industrial track, 2007, pp. 45-52.

[12] P. Kolesar, “A Markovian model for hospital admission scheduling”. Management Science, 16(6), 1970.

[13] J.C. Lowery, J. C. "Design of hospital admissions scheduling system using simulation", In Proceedings of the 28th conference on Winter simulation, IEEE Computer Society, 1996, pp. 1199-1204.

[14] A. Maedche, S. Morana, S Schacht, D Werth, and J. Krumeich, "Advanced User Assistance Systems". Business \& Information Systems Engineering, 58 (5), 2016, pp. 367-370.

[15] D. Min, and Y. Yih, "A simulation study of registration queue disciplines in an outpatient clinic: a two-stage patient flow model", European Journal of Industrial Engineering, 3(2), 2009, pp. 127-145.

[16] B. Mocarzel, D. Shelton, B. Uyan, E. Pérez, J. Jimenez, and L. DePagter, "Modeling and simulation of patient admission services in a multi-specialty outpatient clinic". In Proceedings of the 2013 Winter Simulation Conference: Simulation: Making Decisions in a Complex World, IEEE Press 2013, pp. 2309-2319.

[17] S. Morana, S. Schacht, A. Scherp and A. Maedche, "A review of the nature and effects of guidance design features". Decision Support Systems, 97 (C), 2017, pp. 31-42.

[18] S. Morana, T. Dehling, M. Reuter-Oppermann, and A. Sunyaev, "User Assistance for Health Care Information Systems" SIG-Health Pre-ICIS Workshop, Seoul, South Korea, 2017. https://ssrn.com/abstract=3073261.

[19] S. Nickel, and U.A. Schmidt, "Process improvement in hospitals: a case study in a radiology department”, Quality Management in Healthcare, 18(4), 2009, pp. 326-338.

[20] D. Romanow, C. Sunyoung, and D. Straub, "Riding the Wave: Past Trends and Future Directions for Health IT Research”, MIS Quarterly, Vol. 36 No. 3, 2012.

[21] G. Schmola, and B. Rapp, B. (Eds.) Compliance, Governance und Risikomanagement im Krankenhaus: Rechtliche Anforderungen - Praktische Umsetzung Nachhaltige Organisation, Springer-Verlag, Wiesbaden, 2016.

[22] D. Sundaramoorthi, V.C. Chen, J.M. Rosenberger, S.B. Kim, and D.F. Buckley-Behan, "A data-integrated simulationbased optimization for assigning nurses to patient admissions”, Health care management science, 13(3), 2010, pp. 210-221. 